\title{
Erratum to: Mining clinical text for stroke prediction
}

\author{
Elham Sedghi $^{1} \cdot$ Jens H. Weber $^{1} \cdot$ Alex Thomo $^{1} \cdot$ Maximilian Bibok $^{2}$.
} Andrew M. W. Penn ${ }^{2}$

Published online: 5 October 2015

(C) Springer-Verlag Wien 2015

\section{Erratum to: Netw Model Anal Health Inform \\ Bioinforma (2015) 4:16 \\ DOI 10.1007/s13721-015-0090-5}

In the original publication, the last author's name and Table 2 were incorrect.

The correct name of the last author should be Andrew M. W. Penn. The author's name has been corrected in the original article.

The correct version of Table 2 is given below:

The online version of the original article can be found under doi:10.1007/s13721-015-0090-5.

Elham Sedghi

elham_Sedghi@yahoo.com; esedghi@uvic.ca

Jens H. Weber

jens@uvic.ca

Alex Thomo

thomo@uvic.ca

Maximilian Bibok

Maximilian.Bibok@viha.ca

Andrew M. W. Penn

Andrew.Penn@viha.ca

1 Department of Computer Science, University of Victoria, Victoria, BC, Canada

2 SpecTRA Research Project, Vancouver Island Health Authority, Victoria, BC, Canada 
Table 2 Results using codified data

\begin{tabular}{llllllll}
\hline & Logistic & Naive Bayes & SVM & Neural Network & IBK & J48 & Random Forest \\
\hline Recall (Sensitivitv) & $83.2 \%$ & $76.1 \%$ & $84.0 \%$ & $75.9 \%$ & $76.0 \%$ & $78.7 \%$ & $81.6 \%$ \\
Snecificitv & $63.7 \%$ & $68.7 \%$ & $63.3 \%$ & $72.4 \%$ & $50.5 \%$ & $54.0 \%$ & $55.6 \%$ \\
Precision & $73.0 \%$ & $74.1 \%$ & $73.0 \%$ & $76.4 \%$ & $64.4 \%$ & $66.8 \%$ & $68.4 \%$ \\
F-Measure & $77.8 \%$ & $75.1 \%$ & $78.1 \%$ & $76.1 \%$ & $69.7 \%$ & $72.3 \%$ & $74.4 \%$ \\
ROC & $82.1 \%$ & $79.9 \%$ & $73.7 \%$ & $80.3 \%$ & $63.2 \%$ & $67.5 \%$ & $76.1 \%$ \\
\hline
\end{tabular}

